University of Nebraska - Lincoln

DigitalCommons@University of Nebraska - Lincoln

Faculty Publications from the Department of Engineering Mechanics

Mechanical \& Materials Engineering,

Department of

$7-2003$

\title{
Moving screw dislocations in piezoelectric bimaterials
}

\author{
Xiangfa Wu \\ Department of Engineering Mechanics, University of Nebraska-Lincoln, xfwu@unlserve.unl.edu
}

Yuris A. Dzenis

Department of Engineering Mechanics, University of Nebraska-Lincoln, ydzenis@unl.edu

Wen-Sheng Zou

Beijing Institute of Technology

Follow this and additional works at: https://digitalcommons.unl.edu/engineeringmechanicsfacpub

Part of the Mechanical Engineering Commons

Wu, Xiangfa; Dzenis, Yuris A.; and Zou, Wen-Sheng, "Moving screw dislocations in piezoelectric bimaterials" (2003). Faculty Publications from the Department of Engineering Mechanics. 29. https://digitalcommons.unl.edu/engineeringmechanicsfacpub/29

This Article is brought to you for free and open access by the Mechanical \& Materials Engineering, Department of at DigitalCommons@University of Nebraska - Lincoln. It has been accepted for inclusion in Faculty Publications from the Department of Engineering Mechanics by an authorized administrator of DigitalCommons@University of Nebraska - Lincoln. 
Published in physica status solidi (b) 238:1 (2003), pp. 120-126; doi 10.1002/pssb.200301805

Copyright (C) 2003 WILEY-VCH Verlag GmbH \& Co. KGaA, Weinheim. Used by permission.

http://www3.interscience.wiley.com/journal/40001185/home

Submitted November 18, 2002; revised March 24, 2003; accepted April 2, 2003; published online June 10, 2003.

\title{
Moving screw dislocations in piezoelectric bimaterials
}

\author{
Xiang-Fa Wu, ${ }^{1}$ Yuris A. Dzenis, ${ }^{1}$ and Wen-Sheng Zou ${ }^{2}$ \\ ${ }^{1}$ Department of Engineering Mechanics, Center for Materials Research and Analysis, \\ University of Nebraska-Lincoln, Lincoln, NE 68588-0526, USA \\ ${ }^{2}$ Department of Vehicle Engineering, Beijing Institute of Technology, Beijing 100081, China \\ Corresponding author - Xiang-Fa Wu: xfwu@unlserve.un.edu
}

\begin{abstract}
An explicit solution for a moving screw dislocation in a piezoelectric bimaterial is given by means of complex variables. The electroelastic fields and forces acting on the moving dislocation are obtained in closed form. As special cases, solutions for a moving screw dislocation in a piezoelectric half-plane with a free or rigid surface are obtained explicitly.
\end{abstract}

\section{Introduction}

Due to their intrinsic electromechanical coupling phenomena, piezoelectric materials are finding extensive electromechanical applications in actuators, sensors, and transducers, etc. Piezoelectric materials such as ferroelectric ceramics are brittle and liable to cracking on all scales from domain to device. Defects such as dislocations, micro-cracks, cavities, and inhomogeneous inclusions, etc., may be embedded in piezoelectric materials during manufacturing process and service. Under external electromechanical loadings, the proper functions and service lifetime of piezoelectric devices may be dramatically reduced due to interactions of these defects and resulting macro-cracking. Theoretically, dislocation models play an important role in the study of defects and macro-cracks in solid materials [1-4]. In the past three decades, a number of investigations have been conducted concerning dislocations in piezoelectric materials. To mention a few, Barnett and Lothe [5] first discussed the dislocation-induced electroelastic fields in anisotropic piezoelectric materials by means of the Stroh formalism. Pak [6] determined the forces acting on a screw dislocation in transversely isotropic piezoelectric materials using the generalized Peach-Koehler formula. These results indicate the effect of the material electromechanical coupling property on the electroelastic fields. Recently, further investigations have been performed on a screw dislocation interacting with an elliptical inhomogeneity [7], a free boundary [8] in homogenous piezoelectric materials, and an interface in piezoelectric bimaterials $[9,10]$. In the case of a screw dislocation interacting with cracks, various crack configurations in homogenous piezoelectric materials have been considered such as a semi-infinite crack [11, 12], Griffith crack [13], and so on. Furthermore, detailed investigations have been achieved on the electroelastic fields and the fracture parameters of a screw dislocation interacting with a semi-infinite crack [8, 14], Griffith crack [8], edge crack [8, 15], and wedge crack [16, 17] between two bonded dissimilar piezoelectric materials. These results still keep the fundamental characteristics of dislocation-crack interactions and also indicate the electromechanical coupling and geometric boundary effects on the electroelastic fields and the fracture parameters. In the limiting cases, the above interfacial solutions cover those for homogenous materials. 
Although stationary dislocations in piezoelectric materials have been discussed thoroughly, moving dislocation problems have not yet attracted much attention. The simplest case of a moving screw dislocation in homogenous piezoelectric materials was considered recently [18]. In this paper, we study the fundamental solution of a moving screw dislocation in a piezoelectric bimaterial by means of complex variables. The electroelastic fields and forces acting on the moving dislocation are obtained explicitly. As special cases, solutions for a moving screw dislocation in a piezoelectric half-plane with a free or rigid surface are derived explicitly.

\section{Basic equations}

Consider two dissimilar piezoelectric half-planes bonded along the $x_{1}$-axis, as shown in Figure 1 . The piezoelectric materials are assumed to be transversely isotropic, with the isotropic basal planes parallel to the $\left(x_{1}, x_{2}\right)$-plane and the poling directions along the $x_{3}$-axis.

The dynamic boundary value problem of the piezoelectric materials is constructed assuming only nontrivial out-of-plane mechanical displacement and in-plane electric field such that

$$
\begin{array}{ll}
u_{1}=u_{2}=0, & u_{3}=u_{3}\left(x_{1}, x_{2}, t\right), \\
E_{1}=E_{1}\left(x_{1}, x_{2}, t\right), & E_{2}=E_{2}\left(x_{1}, x_{2}, t\right), \quad E_{3}=0 .
\end{array}
$$

In this case, the constitutive relations become

$$
\sigma_{13}=c_{44} u_{3,1}+e_{15} \phi_{, 1}, \quad D_{1}=e_{15} u_{3,1}-\varepsilon_{11} \phi_{, 1}, \quad \sigma_{23}=c_{44} u_{3,2}+e_{15} \phi_{, 2}, \quad D_{2}=e_{15} u_{3,2}-\varepsilon_{11} \phi_{, 2}
$$

where $\sigma_{k 3}$ is the stress tensor, $D_{k}(k=1,2)$ the electric displacement vector, $c_{44}$ the elastic modulus measured at constant electric field, $e_{15}$ and $\varepsilon_{11}$ the piezoelectric and dielectric constants, and $\phi$ the electric potential.

The electric field is given by

$$
E_{1}=-\phi_{, 1}, \quad E_{2}=\phi_{, 2}
$$

The governing equations are

$$
\sigma_{13,1}+\sigma_{23,2}=\rho \ddot{u}_{3}, \quad D_{1,1}+D_{2,2}=0,
$$

where $\left({ }^{*}\right)=\partial^{2} / \partial^{2} t$, and $\rho$ is the mass density of the related material. Substituting (3) into (5) yields

$$
c_{44} \nabla^{2} u_{3}+e_{15} \nabla^{2} \phi=\rho \ddot{u}_{3}, \quad e_{15} \nabla^{2} u_{3}-\varepsilon_{11} \nabla^{2} \phi=0,
$$

where $\nabla^{2}=\partial^{2} / \partial x_{1}^{2}+\partial^{2} / \partial x_{2}^{2}$ is the Laplace operator.

When $c_{44} \varepsilon_{11}+e_{15}^{2} \neq 0$, the governing equations in (6) can be rewritten as

$$
\nabla^{2} u_{3}=\frac{\rho \varepsilon_{11}}{c_{44} \varepsilon_{11}+e_{15}^{2}} \ddot{u}_{3}, \quad \nabla^{2} \phi=\frac{\rho e_{15}}{c_{44} \varepsilon_{11}+e_{15}^{2}} \ddot{u}_{3} .
$$

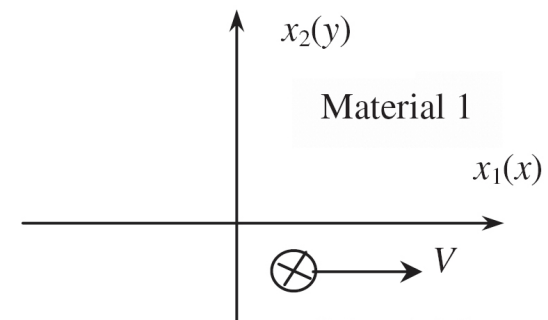

Material 2
Figure 1. Moving screw dislocation in a piezoelectric bimaterial. 
Following Bleustein [19], we introduce an auxiliary function $\varphi$ such that

$$
\varphi=\phi-\frac{e_{15}}{\varepsilon_{11}} u_{3} \text {. }
$$

Substitution of (8) into (7) leads to two decoupled equations as

$$
\nabla^{2} u_{3}=\frac{1}{c^{2}} \ddot{u}_{3}, \quad \nabla^{2} \varphi=0,
$$

where

$$
c=\sqrt{\frac{c_{44} \varepsilon_{11}+e_{15}^{2}}{\rho \varepsilon_{11}}} .
$$

The stresses and electric displacements can be expressed in terms of two independent functions $u_{3}$ and $\varphi$ as

$$
\left[\begin{array}{l}
\sigma_{13} \\
D_{1}
\end{array}\right]=C\left[\begin{array}{l}
u_{3,1} \\
\varphi_{, 1}
\end{array}\right], \quad\left[\begin{array}{l}
\sigma_{23} \\
D_{2}
\end{array}\right]=C\left[\begin{array}{l}
u_{3,2} \\
\varphi_{, 2}
\end{array}\right],
$$

where

$$
C=\left[\begin{array}{cc}
\frac{c_{44} \varepsilon_{11}+e_{15}^{2}}{\varepsilon_{11}} & e_{15} \\
0 & -\varepsilon_{11}
\end{array}\right] .
$$

The analysis presented above shows that the electroelastic solutions for piezoelectric materials subjected to dynamic antiplane mechanical and in-plane electrical loadings can be represented by two independent functions $u_{3}$ and $\varphi$ satisfying the boundary conditions.

\section{Moving screw dislocation in two bonded dissimilar piezoelectric materials}

Consider an infinitely long screw dislocation moving constantly in a piezoelectric bimaterial. Without loss of generality, at time $t=0$, assume the transient location of the screw dislocation $z_{0}\left(z_{0}=x_{10}+i x_{20}, x_{20}\right.$ $<0)$ in the lower half-plane. The dislocation is characterized by a Burgers vector $b$, an electric potential jump $\Delta \phi$, and moving parallel to the $x_{1}$-axis with constant speed $V(V<c)$, as shown in Figure 1 . By means of the superposition scheme [1-3], the overall solution of this problem can be constructed from the solution for a moving screw dislocation in an infinite homogenous piezoelectric medium and an auxiliary potential (interface image) satisfying the interface continuity conditions. The electroelastic solutions, $u_{3}(z)$ and $\phi(z)$, for a moving screw dislocation in an infinite homogenous piezoelectric medium have been obtained by Wang and Zhong [18].

By a coordinate translation, we introduce a new coordinate system $(x, y)$ such that

$$
x=x_{1}-V t, y=x_{2} .
$$

In this new coordinate system, Equation (9) can be rewritten as

$$
\alpha^{2} u_{3, x x}+u_{3, y y}=0, \quad \varphi_{, x x}+\varphi_{, y y}=0,
$$

where

$$
\alpha=\sqrt{1-\frac{V^{2}}{c^{2}}} \quad(V<c) .
$$


The general solutions to Equations (14) can be expressed in terms of two modified holomorphic functions such that

$$
u_{3}=\operatorname{Im}\left[U\left(z_{1}\right)\right], \quad \varphi=\operatorname{Im}[\Phi(z)],
$$

where $z_{1}=x+i \alpha y, z=x+i y$, and $\operatorname{Im}[]$ stands for the imaginary part of an analytic function.

In the new coordinate system, by using the Cauchy-Euler conditions for analytic functions, the stress and electric displacement relations in (11) can be rewritten as

$$
\left[\begin{array}{l}
\sigma_{13} \\
D_{1}
\end{array}\right]=C\left[\begin{array}{l}
u_{3,1} \\
\varphi_{, 1}
\end{array}\right]=C \operatorname{Im}\left[\begin{array}{l}
U^{\prime}\left(z_{1}\right) \\
\Phi^{\prime}(z)
\end{array}\right], \quad\left[\begin{array}{c}
\sigma_{23} \\
D_{2}
\end{array}\right]=C\left[\begin{array}{l}
u_{3,2} \\
\varphi_{, 2}
\end{array}\right]=L \operatorname{Re}\left[\begin{array}{c}
U^{\prime}\left(z_{1}\right) \\
\Phi^{\prime}(z)
\end{array}\right]
$$

where $C$ is defined in (12), $L$ is defined as

$$
L=C\left[\begin{array}{ll}
\alpha & 0 \\
0 & 1
\end{array}\right]=\left[\begin{array}{cc}
\frac{\alpha\left(c_{44} \varepsilon_{11}+e_{15}^{2}\right)}{\varepsilon_{11}} & e_{15} \\
0 & -\varepsilon_{11}
\end{array}\right],
$$

and $\operatorname{Re}[]$ is the real part of an analytic function.

The screw dislocation is defined as

$$
\oint_{\Gamma z_{0}} \mathrm{~d} u_{3}=b, \quad \oint_{\Gamma z_{0}} \mathrm{~d} \phi=\Delta \phi
$$

or in the form of $u_{3}$ and $\varphi$ as

$$
\oint_{\Gamma z_{0}} \mathrm{~d} u_{3}=b, \quad \oint_{\Gamma z_{0}} \mathrm{~d} \varphi=\Delta \phi-\frac{e_{15}}{\varepsilon_{11}} b,
$$

where $\Gamma$ is a closed contour surrounding the dislocation core $z=z_{0}$ in the physical plane.

The electroelastic solutions $U\left(z_{1}\right)$ and $\Phi(z)$ for a moving screw dislocation with constant speed $V$ parallel to the $x_{1}$-axis in an infinite homogenous piezoelectric medium can be expressed as [18]

$$
U\left(z_{1}\right)=\frac{b}{2 \pi} \ln \left(z_{1}-z_{10}\right), \quad \Phi(z)=\frac{\varepsilon_{11} \Delta \phi-e_{11} b}{2 \pi \varepsilon_{11}} \ln \left(z-z_{0}\right),
$$

where $z_{10}=x_{0}+i \alpha y_{0}$ and $z_{0}=x_{0}+i y_{0}$.

Now let us construct the solution for the current bimaterial problem. In the following procedure, indices 1 and 2 denote material constants, displacements, electric potentials, stresses, and electric displacements of the upper and lower half-planes, respectively. The $x_{1}(\mathrm{x})$-axis is directed along the interface, as shown in Figure 1. The electroelastic solutions $U\left(z_{1}\right)$ and $\varphi(z)$ can be expressed as

$$
U(z)= \begin{cases}U_{1}\left(z_{1}\right), & \left.z \in D_{1} \text { (i.e., } y>0\right), \\ U_{2}\left(z_{1}\right)+U_{0}\left(z_{1}\right), & \left.z \in D_{2} \text { (i.e., } y<0\right),\end{cases}
$$

and

$$
\Phi(z)= \begin{cases}\Phi_{1}(z), & \left.z \in D_{1} \text { (i.e., } y>0\right), \\ \Phi_{2}(z)+\Phi_{0}(z), & \left.z \in D_{2} \text { (i.e., } y<0\right) .\end{cases}
$$

Here $U_{1}\left(z_{1}\right), U_{2}\left(z_{1}\right), \Phi_{1}(z)$, and $\Phi_{2}(z)$ are unknown analytic functions to be determined, and $U_{0}\left(z_{1}\right)$ and $\Phi_{0}(z)$ are solutions for a moving screw dislocation in an infinite homogenous piezoelectric material as expressed in (21) with material constants related to the lower half-plane. 
In order to simplify the derivation, we introduce the five vectors

$$
f_{1}(z)=\left[\begin{array}{l}
U_{1}\left(z_{1}\right) \\
\Phi_{1}(z)
\end{array}\right], \quad f_{2}(z)=\left[\begin{array}{l}
U_{2}\left(z_{1}\right) \\
\Phi_{2}(z)
\end{array}\right],
$$

and

$$
u=\left[\begin{array}{l}
u_{3} \\
\phi
\end{array}\right], \quad t=\left[\begin{array}{l}
\sigma_{23} \\
D_{2}
\end{array}\right] .
$$

With the aid of (8), (11), and (16), (25) can be rewritten as

$$
\boldsymbol{u}=B \operatorname{Im}\left\{[U, \Phi]^{T}\right\}=-i B / 2\{[\boldsymbol{f}(z)-\overline{\boldsymbol{f}}(z)]\}
$$

and

$$
t=L / 2\left[f^{\prime}(z)+\bar{f}^{\prime}(z)\right],
$$

where

$$
B=\left[\begin{array}{cc}
1 & 0 \\
e_{15} / \varepsilon_{11} & 1
\end{array}\right]
$$

and $\left(^{-}\right)$denotes the conjugate of an analytic function.

Now let us determine the unknown $U_{1}\left(z_{1}\right), U_{2}\left(z_{1}\right), \Phi_{1}(z)$, and $\Phi_{2}(z)$ by enforcing continuity of the outof-plane displacement, electric potential, mechanical force, and electric displacement across the interface $\left(x_{2}=0\right)$. It should be noted that $U_{1}\left(z_{1}\right)$ and $\Phi_{1}(z)$ are analytic in the upper half-plane, while $U_{2}\left(z_{1}\right)$ and $\Phi_{2}(z)$ are analytic in the lower half-plane. By using the relations (24)-(27), the continuity of the out-ofplane displacement and electric potential across the interface requires

$$
B_{1}\left[f_{1}(x)-\overline{\boldsymbol{f}}_{1}(x)\right]=B_{2}\left[\boldsymbol{f}_{2}(x)-\overline{\boldsymbol{f}}_{2}(x)+\boldsymbol{f}_{0}(x)-\overline{\boldsymbol{f}}_{0}(x)\right] .
$$

Rearranging (29), we obtain

$$
B_{1} f_{1}(x)+B_{2} \bar{f}_{2}(x)-B_{2} f_{0}(x)=B_{2} f_{2}(x)+B_{1} \bar{f}_{1}(x)-B_{2} \bar{f}_{0}(x) .
$$

Equation (30) holds along the whole x-axis. Moreover, the functions on the left-hand side are analytic in the upper half-plane, while those on the right-hand side are analytic in the lower half-plane. With the standard analytic continuity arguments, we have

$$
B_{1} f_{1}(z)+B_{2} \bar{f}_{2}(z)-B_{2} f_{0}(z)=0, \quad z \in D_{1} .
$$

With the same arguments, the stress and electric force continuity across the interface leads to

$$
L_{1} \boldsymbol{f}_{1}^{\prime}(z)-L_{2} \bar{f}_{2}^{\prime}(z)-L_{2} \boldsymbol{f}_{0}^{\prime}(z)=0, \quad z \in D_{1} .
$$

Relations (31) and (32) yield

$$
\begin{aligned}
& f_{1}(z)=2\left(B_{2}^{-1} B_{1}+L_{2}^{-1} L_{1}\right)^{-1} f_{0}(z), \quad z \in D_{1}, \\
& f_{2}(z)=\left(I+L_{2}^{-1} L_{1} B_{1}^{-1} B_{2}\right)^{-1}\left(I-L_{2}^{-1} L_{1} B_{1}^{-1} B_{2}\right)^{-1} \bar{f}_{0}(z), \quad z \in D_{2},
\end{aligned}
$$

where $I$ is a second-order identity matrix, and the subscripts 1 and 2 behind $B$ and $L$ stand for the material matrices corresponding to the upper and lower half-planes, respectively.

In the above, by letting $L_{1}=L_{2}$ and $B_{1}=B_{2}$, results in (33) cover the known solutions in (21). Furthermore, the moving screw dislocation in a half-plane $(y<0)$ with a traction-free and electrically imperme- 
able surface, $y=0$, can be described by

$$
\boldsymbol{f}(z)=\boldsymbol{f}_{0}(z)-\boldsymbol{f}_{0}(\bar{z}), \quad z \in D_{2} .
$$

Another interesting case is the moving screw dislocation in the half-plane $(y<0)$ with a rigid and electrically impermeable surface, $y=0$. The corresponding solutions are

$$
U(z)=U_{0}(z)+U_{0}(\bar{z}), \quad \Phi(z)=\Phi_{0}(z)-\Phi_{0}(\bar{z}), \quad z \in D_{2}
$$

\section{Electroelastic fields}

The electroelastic fields excited by the moving screw dislocation can be obtained by substituting (22), (23), (24), and (33) into (17) as

$$
\begin{aligned}
& {\left[\begin{array}{l}
\sigma_{13} \\
D_{1}
\end{array}\right]=C_{1}\left(B_{2}^{-1} B_{1}+L_{2}^{-1} L_{1}\right)^{-1}\left[\begin{array}{l}
\frac{b}{\pi} \frac{-\sqrt{1-V^{2} / c^{2}}\left(y-y_{0}\right)}{\left(x-x_{0}-V t\right)^{2}+\left(1-V^{2} / c^{2}\right)\left(y-y_{0}\right)^{2}} \\
\frac{\varepsilon_{11} \Delta \phi-e_{11} b}{\pi \varepsilon_{11}} \frac{-\left(y-y_{0}\right)}{\left(x-x_{0}-V t\right)^{2}+\left(y-y_{0}\right)^{2}}
\end{array}\right],} \\
& {\left[\begin{array}{l}
\sigma_{23} \\
D_{2}
\end{array}\right]=L_{1}\left(B_{2}^{-1} B_{1}+L_{2}^{-1} L_{1}\right)^{-1}\left[\begin{array}{l}
\frac{b}{\pi} \frac{x-x_{0}-V t}{\left(x-x_{0}-V t\right)^{2}+\left(1-V^{2} / c^{2}\right)\left(y-y_{0}\right)^{2}} \\
\frac{\varepsilon_{11} \Delta \phi-e_{11} b}{\pi \varepsilon_{11}} \frac{x-x_{0}-V t}{\left(x-x_{0}-V t\right)^{2}+\left(y-y_{0}\right)^{2}}
\end{array}\right]}
\end{aligned}
$$

in the upper half-plane and

$$
\begin{aligned}
{\left[\begin{array}{l}
\sigma_{13} \\
D_{1}
\end{array}\right]=} & C_{2}\left[\left(I+L_{2}^{-1} L_{1} B_{1}^{-1} B_{2}\right)^{-1}\left(I-L_{2}^{-1} L_{1} B_{1}^{-1} B_{2}\right)-I\right] \\
& \times\left[\begin{array}{l}
\frac{b}{\pi} \frac{-\sqrt{1-V^{2} / c^{2}}\left(y-y_{0}\right)}{\left(x-x_{0}-V t\right)^{2}+\left(1-V^{2} / c^{2}\right)\left(y-y_{0}\right)^{2}} \\
\frac{\varepsilon_{11} \Delta \phi-e_{11} b}{\pi \varepsilon_{11}} \frac{-\left(y-y_{0}\right)}{\left(x-x_{0}-V t\right)^{2}+\left(y-y_{0}\right)^{2}}
\end{array}\right], \\
{\left[\begin{array}{l}
\sigma_{23} \\
D_{2}
\end{array}\right]=} & L_{2}\left[\left(I+L_{2}^{-1} L_{1} B_{1}^{-1} B_{2}\right)^{-1}\left(I-L_{2}^{-1} L_{1} B_{1}^{-1} B_{2}\right)+I\right] \\
\times & {\left[\begin{array}{l}
\frac{b}{\pi} \frac{x-x_{0}-V t}{\left(x-x_{0}-V t\right)^{2}+\left(1-V^{2} / c^{2}\right)\left(y-y_{0}\right)^{2}} \\
\frac{\varepsilon_{11} \Delta \phi-e_{11} b}{\pi \varepsilon_{11}} \frac{x-x_{0}-V t}{\left(x-x_{0}-V t\right)^{2}+\left(y-y_{0}\right)^{2}}
\end{array}\right] }
\end{aligned}
$$

in the lower half-plane, where $B_{j}, C_{j}$ and $L_{j}(j=1,2)$ are the material matrices defined in (12), (18), and (28). By letting $B_{1}=B_{2}, C_{1}=C_{2}$, and $L_{1}=L_{2}$, the above solutions cover those in the literature [18].

From the electroelastic solutions (36)-(39), it is found that the electroelastic solutions are dependent on the ratios $B_{2} / B_{1}$ and $L_{2} / L_{1}$ When the electromechanical properties of two component materials are highly mismatched, the electroelastic fields excited by the moving screw dislocation are modified significantly, in which two limiting cases are the moving screw dislocation in a half-plane with rigid or free surface as discussed in Section 3. 
The above solutions also cover the static ones by letting the dislocation speed $V \rightarrow 0$. Furthermore, if letting $e_{15}=0$ and $\Delta \phi=0$, the aforementioned solutions will reduce to those for a moving screw dislocation in two bonded dissimilar elastic media.

The image forces acting on the screw dislocation can be obtained straightforwardly using the generalized Peach-Koehler formula [6] and the whole electroelastic field solutions (36)-(39).

\section{Conclusion}

The electroelastic solutions for a moving screw dislocation in a piezoelectric bimaterial are obtained in this paper. The obtained solutions indicate the effects of material mismatch and coupling electromechanical property on the electroelastic fields. These fundamental solutions can be further used as the Green's functions to solve the dynamic electroelastic problems in piezoelectric materials with defects. The given solutions can be also used to determine the dynamic electroelastic fields of defects in a piezoelectric/nonpiezoelectric material/strip bonded to another piezoelectric/non-piezoelectric material/strip, which is very common in piezoelectric applications such as acoustic sensors.

\section{Acknowledgments}

The authors would like to thank the anonymous reviewers from physica status solidi (b) for their careful reading and helpful comments for the improvement of this paper. X.-F. Wu would like to express his grateful acknowledgement to the Milton E. Mohr Research Fellowship of the Engineering College at the University of Nebraska-Lincoln, 2001, 2002.

\section{References}

[1] R. Thomsom, Solid State Phys. 39, 1 (1986).

[2] Z. Suo, Int. J. Solids Struct. 25, 1133 (1989).

[3] Z. Suo, Proc. R. Soc. Lond. A 427, 331 (1990).

[4] J. Weertman, Dislocation Based Fracture Mechanics (World Scientific, Singapore, 1996).

[5] D. M. Barnett and J. Lothe, phys. stat. sol. (b) 67, 105 (1975).

[6] Y. E. Pak, J. Appl. Mech. (ASME) 57, 863 (1990).

[7] S. A. Meguid and W. Deng, Int. J. Solids Struct. 35, 1467 (1998).

[8] X. F. Li and X. Y. Duan. phys. stat. sol. (b) 227, 613 (2001).

[9] J. X. Liu, S. Y. Du, and B. Wang, Mech. Res. Commun. 26, 415 (1999).

[10] X. F. Wu, S. Cohn, and Y. A. Dzenis, Int. J. Eng. Sci. 41, 667 (2003).

[11] K. Y. Lee, W. G. Lee, and Y. E. Pak, J. Appl. Mech. (ASME) 67, 165 (2000).

[12] B. J. Chen, Z. M. Xiao, and K. M. Liew, Int. J. Solids Struct. 39, 1505 (2002).

[13] K. K. Kwon and K. Y. Lee, J. Appl. Mech. (ASME) 69, 55 (2002).

[14] A. K. Soh, J. X. Liu, and D. N. Fang, phys. stat. sol. (b) 232, 273 (2002).

[15] X. F. Li and T. Y. Fan, Arch. Appl. Mech. 71, 703 (2001).

[16] B. J. Chen, Z. M. Xiao, and K. M. Liew, Int. J. Eng. Sci. 40, 1665 (2002).

[17] X. F. Wu, Y. A. Dzenis, and W. S. Zou, Int. J. Fract. 117, L9 (2002).

[18] X. Wang and Z. Zhong, Mech. Res. Commun. 29, 425 (2002).

[19] J. L. Bleustein, Appl. Phys. Lett. 13, 412, (1969). 